\title{
The Elephant in the Room. Über das Verhältnis von demographischem Wandel, Daseinsvorsorge und zivilgesellschaftlichem Engagement in Deutschland
}

\author{
Robert Nadler ${ }^{1}$ iD
}

Eingegangen: 18. April 2017 / Angenommen: 20. September 2017 / Online publiziert: 4. Oktober 2017

(c) Springer-Verlag GmbH Deutschland 2017

Zusammenfassung Das Verhältnis von demographischem Wandel, Daseinsvorsorge und zivilgesellschaftlichem Engagement wird in der deutschen Raumplanung häufig als Substitutionslogik für staatliche Erbringung von Daseinsvorsorgeleistungen durch zivilgesellschaftliche Akteure entworfen. Ziel dieses Beitrags ist es, diese Substitutionslogik kritisch zu betrachten. Der Artikel geht der Frage nach, wie realistisch eine solche Substitution vor dem Hintergrund des demographischen Wandels eigentlich ist. Anhand eines Literaturüberblicks findet eine Begriffsbestimmung zum zivilgesellschaftlichen Engagement sowie eine Beschreibung der empirischen Ausprägung des Engagements in Deutschland statt. Anschließend werden die bestehenden diskursiven Verbindungen zwischen Daseinsvorsorge und demographischem Wandel in der deutschen Raumplanung skizziert. Zentraler Befund des Beitrags ist die Ableitung von drei Dilemmata (geographisches, politisches und motivationales Dilemma), die einer realistischen Umsetzung von Substitution entgegenstehen. Im Ergebnis werden Vorschläge zu einer Neugestaltung der Verbindungslinien zwischen Daseinsvorsorge und zivilgesellschaftlichem Engagement im Kontext des demographischen Wandels präsentiert. Es wird argumentiert, dass neben einer stärkeren Anerkennungskultur für Engagement auch ein raumdifferenzierender Ansatz der Substitution notwendig ist und staatliche Akteure ihr Selbstverständnis und ihr Verhältnis zu zivilgesellschaftlichen Gruppen mit dem Ziel einer gleichberechtigten Partnerschaft überdenken müssen, um die Substitutionslogik

Dr. Robert Nadler

robert.nadler@ils-forschung.de

1 Forschungsgruppe Alltagsmobilität und Verkehrssysteme, ILS - Institut für Landes- und Stadtentwicklungsforschung, Brüderweg 22-24, 44135 Dortmund, Deutschland zumindest in Teilen als realistischen Ansatz für eine demographieresiliente Bereitstellung von Daseinsvorsorge nutzen zu können.

Schlüsselwörter Zivilgesellschaftliches Engagement . Daseinsvorsorge · Demographischer Wandel

\section{The Elephant in the Room. On the Relation of Demographic Change, Public Services and Civic Engagement in Germany}

Abstract In Germany's territorial planning community, the relation of demographic change, the provision of public services and civic engagement is mainly sketched out as a substitution logic, in which volunteer work substitutes for employed work of state agencies in the provision of public services. The aim of this paper is a critical assessment of this substitution logic. The central question is: how realistic is this fictional model of substitution in the light of current patterns of demographic change in Germany? Methodologically the paper draws on a literature review, which is, first, used to outline definitions and empirical evidence regarding civic engagement in Germany, and second, to present in detail and critically reflect the main discourse in German territorial planning regarding the relation of public services and demographic change. The central result is the description of three dilemmas (geographic, political and motivational dilemma), that hinder a realistic implementation of substitution. As a consequence, suggestions are made for a better conceptualization of the relation of public service provision and civic engagement in the light of demographic change. It is argued that civic engagement should more strongly be conceived in combination with tools for recognition, the geographic disparities of civic engagement must be consid- 
ered more strongly, and state agents must reflect on and redefine their role and self-conception in relation to engaging social groups, with the aim of a more equal partnership.

Keywords Civic Engagement · Public Services · Demographic Change

\section{Einleitung}

Die Bevölkerung in Deutschland entwickelt sich auf kleinräumiger Ebene durchaus gegensätzlich. Weiterhin wachsende Großstadtregionen stehen flächenhafter Schrumpfung in ohnehin schon dünn besiedelten Regionen gegenüber. In letzteren stehen die vorhandenen Strukturen und Standards der Daseinsvorsorge durch den demographischen Wandel unter erheblichem Anpassungsdruck. Neben Bevölkerungsrückgang sorgen Abwanderung und Alterung für eine sich verändernde Nachfrage nach technischen und sozialen Infrastrukturen sowie staatlichen Dienstleistungen. Gelingt es hier nicht, die Daseinsvorsorge neu zu gestalten, droht diesen Regionen eine Abwärtsspirale aus weiterer Abwanderung und ungesteuertem Rückbau von Infrastrukturen. Dabei ist die öffentliche Hand einem Spardruck ausgesetzt, in dessen Konsequenz bestehende Angebote ausgedünnt oder komplett eingestellt werden. Zudem ist in schrumpfenden Regionen für bestimmte Daseinsvorsorgebereiche mittlerweile die kritische Masse an Nachfragern nicht mehr vorhanden und Versuche der Privatisierung laufen ins Leere, da die Privatwirtschaft - ohne eine wirtschaftlich tragfähige Nachfrage - oft nicht mehr an einer Übernahme vormals staatlicher Leistungen interessiert ist.

Die Aktivierung zivilgesellschaftlichen Engagements wird in diesem Zusammenhang als ein ,potenter Notnagel“ verstanden (Monheim 2005: 65), um in dünn besiedelten Räumen infrastrukturelle Defizite auszugleichen, bestehende Angebote zu erhalten und zu verbessern oder innovative Sonderlösungen zu entwickeln (Kocks 2005; Monheim 2005). Eine differenzierte Auseinandersetzung mit den Chancen, Risiken und Grenzen dieser - wie ich es nennen werde - ,Substitutionslogik' steht jedoch weitgehend aus. In diesem Beitrag umreiße ich die derzeit bestehenden Probleme und Grenzen dieser Strategie und möchte damit zur kritischen Reflexion anregen. Ich gehe der zentralen Frage nach, ob eine Kompensierung vormals staatlich erbrachter Daseinsvorsorgeleistungen durch zivilgesellschaftliches Engagement eine tragfähige Lösung zur Abmilderung negativer Auswirkungen des demographischen Wandels darstellt. Dazu wird im nächsten Kapitel ein Überblick über die Definitionsversuche von zivilgesellschaftlichem Engagement und empirische Befunde zum Engagement in Deutschland gegeben. Das dritte Kapitel skizziert bestehende diskursive Verbindungslinien zwischen Daseinsvorsor- ge, demographischem Wandel und zivilgesellschaftlichem Engagement in Deutschland, in denen sich die Substitutionslogik artikuliert. Im vierten Kapitel findet eine kritische Auseinandersetzung mit dieser Substitutionslogik statt. Abschließend werden die Erkenntnisse im Hinblick auf zukünftige Forschungsbedarfe zusammengefasst.

\section{Zivilgesellschaftliches Engagement in Deutschland}

\subsection{Begriffsbestimmung, ,Zivilgesellschaftliches Engagement"6}

Der Begriff der Zivilgesellschaft beschreibt laut Westle und Gabriel (2008: 16) ein vermittelndes „Netz intermediärer Organisation zwischen der Makro-Ebene des Staates und der Wirtschaftsstruktur [sowie] der Mikro-Ebene des Individuums“. Adloff (2005: 8) erkennt drei kennzeichnende Elemente der Zivilgesellschaft: erstens gesellschaftliche Organisationen und Institutionen, zweitens zivile Umgangsformen (in der Regel Toleranz, Gewaltfreiheit, Verständigung sowie staatlicher Schutz der Meinungs-, Versammlungs- und Pressefreiheit) sowie drittens als utopisches Projekt das selbstregierte, demokratische Zusammenleben. Typisch für das erste Element sind Vereine, Verbände und soziale Bewegungen. Diese seien zu verstehen als ,ein gesellschaftlicher Raum, [welcher] die plurale Gesamtheit der öffentlichen Assoziationen, Vereinigungen und Zusammenkünfte [umfasst], die auf dem freiwilligen Zusammenhandeln der Bürger und Bürgerinnen beruht" (Adloff 2005: 8). Die Zivilgesellschaft wird damit als Teilbereich der Gesamtgesellschaft verstanden. Vom Staat ist sie abgegrenzt, da sie keine direkte politische Macht ausübt. Von der Wirtschaft unterscheidet sie sich, da keine private Aneignung von Arbeit und Produktion erfolgt, und von der Familie wird sie durch die prinzipielle Offenheit für jedermann unterschieden. Zwischen diesen Gesellschaftsteilen bestehen aber fließende Übergänge (Pollack 2004: 27 f.).

Nach Putnams Verständnis (Putnam 1993; Putnam 2000) ist die Zivilgesellschaft in Form von Netzwerken zivilen Engagements, neben dem sozialen Vertrauen und Reziprozitätsnormen, ein Bestandteil von Sozialkapital (vgl. auch Kern 2004). Kritisch wird diese Verwendung des Sozialkapital-Konzeptes im Zusammenhang mit Zivilgesellschaft von Mayer (2005: 592 f.) gesehen. Es lenke von den Ursachen sozialräumlicher Ausgrenzungsprozesse ab und führe dazu, dass die Zivilgesellschaft genutzt werden könne, um neoliberale Restrukturierungsprozesse zu verankern, Auswirkungen von Privatisierung abzufangen und wohlfahrtsstaatliche Leistungen zu ersetzen.

Sobald sich Bürgerinnen und Bürger mit ihrer Aktivität im Zwischenbereich zwischen Familie, Staat und 
Wirtschaft bewegen, wird also von zivilgesellschaftlichem - oder bürgerschaftlichem - Engagement gesprochen. Laut Deutschem Bundestag (2002: 24 ff.) sind unter bürgerschaftlichem Engagement freiwillige, unbezahlte und gemeinwohlorientierte Tätigkeiten zu verstehen, die gemeinschaftlich durchgeführt werden und in der Öffentlichkeit stattfinden. Dieses Verständnis von bürgerschaftlichem Engagement vertritt auch die Bundesarbeitsgemeinschaft der Freiwilligenagenturen (BAGFA 2014: 2): „Bürgerschaftliches Engagement bedeutet für uns unentgeltliches Mitwirken und Mitgestalten, Übernehmen von Verantwortung für gesellschaftliche Belange in allen Bereichen sowie Einbringen und Realisieren von individuellen Ideen und Projekten." Weiterhin führt die BAGFA aus, dass bürgerschaftliches Engagement den Charakter einer freiwilligen Zeitspende hat und den Engagierten natürlich die für sie aus dem Engagement entstehenden Kosten erstattet werden können, jedoch darüber hinaus „Anerkennungsformen nur in begrenztem Umfang als geldwerte Leistungen“" erfolgen sollen (BAGFA 2014: 5).

Andere Autoren haben unterschiedliche Begriffsverständnisse. Laut Mai und Swiaczny (2008) kann bürgerschaftliches Engagement dem Begriff des ehrenamtlichen Engagements gegenübergestellt werden. So wird unter ehrenamtlichem Engagement bzw. Ehrenamt häufig eine unentgeltliche, auf das Gemeinwohl abzielende und nicht an beruflichen Qualifikationen orientierte Tätigkeit innerhalb bestehender zivilgesellschaftlicher Institutionen (z. B. von Vereinen und Verbänden) verstanden. Diesem eher traditionellen Begriff des Ehrenamtes werden im Zuge gesellschaftlicher Individualisierung die Begriffe bürgerschaftliches bzw. freiwilliges Engagement gegenübergestellt, die Tätigkeiten außerhalb etablierter Institutionen umfassen können. Zudem wird mit freiwilligem Engagement auch die Orientierung am individuellen Eigennutzen für die Aktiven betont. Laut Priller (2010: 200) ist aufgrund der verschiedenen Verständnisse der Begriff des zivilgesellschaftlichen Engagements am besten geeignet, die verschiedenen Formen von Engagement abzubilden. Da mein Beitrag nicht primär die Begriffsklärung verfolgt, werde ich dieser Einschätzung Prillers folgend ebenfalls von zivilgesellschaftlichem Engagement sprechen.

\subsection{Empirische Befunde zum zivilgesellschaftlichen Engagement in Deutschland}

Als Gründe dafür, sich über zivilgesellschaftliches Engagement in der Mitgestaltung des gesellschaftlichen Lebens einzubringen, identifiziert Wade (2015: $136 \mathrm{ff}$.) die persönliche Betroffenheit der Bürgerinnen und Bürger hinsichtlich bestimmter Themen des stadtgesellschaftlichen Miteinanders, die Existenz persönlicher Kontakte und Verbindungen im Viertel (Sozialkapital), den persönlichen Bezug und Ge- staltungswillen bezüglich des eigenen Wohn- und Lebensumfeldes, die Bewusstseinsarbeit und Aufklärung anderer Bewohner bezüglich sozialer Ungleichheiten innerhalb der Stadtgesellschaft, die Unterstützung Hilfebedürftiger und den Wunsch nach Solidarität sowie die Schaffung von Gemeinsamkeit und ,Gemeinsinn".

Zivilgesellschaftliches Engagement und die Bereitschaft, ehrenamtlich tätig zu werden, sind in Deutschland regional ungleich verteilt (Simonson/Ziegelmann/Vogel et al. 2017). So lassen sich ein ausgeprägtes West-Ost-Gefälle und ein etwas schwächer ausgeprägtes Süd-Nord-Gefälle hinsichtlich des Bevölkerungsanteils der Engagierten feststellen. Im ersten Engagementbericht des Bundesministeriums für Familie, Senioren, Frauen und Jugend (BMFSFJ) (Enste/ Neumann/Schare 2012: 13) wird darauf hingewiesen, dass das zivilgesellschaftliche Engagement in Ostdeutschland hier sind $31 \%$ der über 14-Jährigen engagiert - geringer ausgeprägt ist als in Westdeutschland (37\%). Auch im Engagementatlas 2009 des Generali Zukunftsfonds verdeutlicht sich ein ähnliches Bild. Hier zeigen Prognos und Generali (2009: 20), dass die ostdeutschen Kreise und kreisfreien Städte weniger zivilgesellschaftliches Engagement verzeichnen können als die westdeutschen Regionen. Insbesondere in den am stärksten schrumpfenden Landkreisen in Deutschland ist der Anteil der zivilgesellschaftlich Engagierten mit am geringsten. Diese regionalen Unterschiede werden mit unterschiedlichen Rahmenbedingungen, etwa der Marginalisierung und Instrumentalisierung der Zivilgesellschaft in der DDR-Zeit, der seit der Wiedervereinigung in den neuen Ländern höheren Arbeitslosigkeit und selektiven Abwanderung junger Bevölkerungsgruppen erklärt (Prognos/Generali 2009: 23). Auch die Struktur der ehrenamtlichen Tätigkeit scheint sich zu unterscheiden eher formell in Vereinsstrukturen im Westen, eher informell in weit verzweigten Netzwerken gegenseitiger Hilfeleistung im Osten (Reim/Schmithals 2008: 78).

Zudem engagieren sich einzelne Bevölkerungsgruppen unterschiedlich stark (Simonson/Ziegelmann/Vogel et al. 2017). Enste, Neumann und Schare (2012: 13) betonen, dass Personen mit einer Zuwanderungsgeschichte seltener längerfristig engagiert sind. Sie erklären dies mit sozialstrukturellen Merkmalen. So haben Engagierte tendenziell höhere Einkommen und höhere Bildungsniveaus. Darüber hinaus sind Frauen seltener engagiert als Männer. Hierzu beschreiben Enste, Neumann und Schare (2012: 13), dass Frauen gerade in Leitungspositionen sowie in auf Sport bezogenem Ehrenamt unterrepräsentiert sind, und sie erklären dies mit der immer noch ungleich verteilten Verantwortung für die Hausarbeit. So hätten junge Mütter, die in der Familiengründungsphase auch ihren Berufseinstieg organisieren müssten, deutlich weniger Zeit für zivilgesellschaftliches Engagement als junge Väter. Bezüglich des Engagements Jugendlicher schlussfolgern Enste, Neumann und Schare 
(2012: 13 f.), dass der „Rückgang des Engagements Jüngerer [...] mit der zunehmenden Mobilität, der Zeitknappheit und den steigenden Anforderungen an den Berufseinstieg erklärt“ werden kann. Gleichzeitig sind „Motive Jugendlicher und junger Erwachsener [...] heute häufiger mit der Erwartung verbunden, aus dem Engagement einen Vorteil für berufliche Kontexte zu ziehen, als dies noch vor zehn Jahren der Fall war“, so Enste, Neumann und Schare (2012: $13 \mathrm{f}$.).

Angesichts des demographischen Wandels lässt sich bundesweit beobachten, dass Engagierte seltener als früher langfristige Verbindlichkeiten eingehen wollen oder können (Enste/Neumann/Schare 2012: 15): „Veränderungen erfährt das bürgerschaftliche Engagement derzeit auch bei der Zusammenarbeit von Haupt- und Ehrenamtlichen sowie bei der Gewinnung von Funktionsträgerinnen und -trägern auf Vorstands- und Führungsebene. In einigen Bereichen des organisierten Engagements sinkt seit Jahren die Bereitschaft, Leitungsfunktionen oder Vorstandspositionen zu übernehmen. Gleichzeitig nehmen kurzfristige oder unregelmäßigere Engagementformen in den letzten zehn Jahren zu." Kritisch sehen viele Engagierte - zumindest laut einer Befragung in Sachsen -, dass die ehrenamtlichen Projekte häufig nicht ausreichend mit finanziellen Mitteln ausgestattet sind. Zunehmend werden auch eine verbesserte Bereitstellung von Räumen und Sachmitteln sowie eine unbürokratische Möglichkeit der Erstattung von Kosten gefordert (SMS 2005: 22).

Hinsichtlich der Themen und Inhalte des Engagements zeigt sich neben der Freiwilligen Feuerwehr seit Jahren ein klarer Fokus sowohl auf Sport und Bewegungsangebote, wodurch insbesondere Männer in das Ehrenamt gebracht werden, sowie Angebote für ältere Bürgerinnen und Bürger (Sittler 2015: 9). Prognos und Generali (2009: 11) konnten zudem aufzeigen, dass Frauen sich stärker in den Bereichen Kinder und Jugend, Kirche und Religion sowie Politik und Interessenvertretung engagieren. Jugendliche zwischen 16 und 18 Jahren engagieren sich am stärksten im Bereich Sport und Freizeit (Prognos/Generali 2009: 11).

Auch Veränderungen auf dem Arbeitsmarkt haben zu weitreichenden Folgen für das zivilgesellschaftliche Engagement geführt. So kommt eine Auswertung des Deutschen Gewerkschaftsbundes (DGB 2016) zu dem Ergebnis, dass die berufliche Mobilität in Deutschland seit Jahren zunimmt und damit das Zeitbudget für Freizeitaktivitäten sinkt. Gerade das Langstreckenpendeln und damit einhergehende multilokale Lebensweisen verringern die Ressourcen vieler Bürgerinnen und Bürger für zivilgesellschaftliches Engagement in der Heimat.

Zukünftig werden sich die Engagementinhalte und -strukturen in Deutschland insbesondere durch die anhaltende Alterung der Gesellschaft, die sinkende Anzahl der Kinder und Jugendlichen, die steigenden Erwerbstäti- genquoten angesichts von Fachkräfteengpässen, anhaltende Wanderung in die großstädtischen Zentren und die sich weiterhin einschränkenden finanziellen Handlungsspielräume der Kommunen verändern (Deutscher Bundestag 2016). Damit werden gerade Bürgerinnen und Bürger in ländlichen Gebieten und Klein- und Mittelstädten stärker selbst organisiert und selbstverantwortlich leben müssen (vgl. Prognos/Generali 2009: 30).

Voraussetzungen für das Entstehen und den Fortbestand einer aktiven Zivilgesellschaft sind ein Kern von überdurchschnittlich engagierten Akteuren, eine kooperationswillige Gemeindeverwaltung und eine lokale „Kultur der Eigenverantwortung" (Neu 2007: 36). Neben erwünschten Effekten wie einer Stärkung der Identifikation mit dem Heimatort sind auch unerwünschte Folgen des Rückzugs des Staates denkbar. Ein Beispiel wäre die von rechtsextremen Parteien und Vereinigungen verfolgte Strategie, über das Organisieren von Freizeitangebote oder das Initiieren von Bürgerinitiativen einen „Weg in die Mitte der Gesellschaft“ zu finden (Heinrich 2005: 34 f.).

Es lässt sich festhalten, dass zivilgesellschaftliches Engagement den Charakter einer freiwilligen Zeitspende von Bürgerinnen und Bürgern hat, die zunehmend flexibler und unverbindlicher wird und in Deutschland regional ungleich verteilt ist. Demnach ist also nicht davon auszugehen, dass zivilgesellschaftliches Engagement in Zukunft automatisch und flächendeckend eine stärkere Rolle in der Ausgestaltung des gesellschaftlichen Miteinanders einnehmen wird. Gleichzeitig wird zivilgesellschaftliches Engagement verstärkt als Ressource für die Erbringung von Leistungen der Daseinsvorsorge diskutiert, wie ich im folgenden Abschnitt darstelle.

\section{Daseinsvorsorge in der Diskussion}

\subsection{Daseinsvorsorge und demographischer Wandel}

Der Begriff der Daseinsvorsorge ist in der Literatur sowie in den gesetzlichen Regelungen des Bundes und der Länder nicht einheitlich definiert. Entsprechend variiert das Verständnis darüber, welche Leistungen darunter $\mathrm{zu}$ verstehen sind (vgl. BMVBS 2013). In Anlehnung an die EU-Arbeitsbegriffe differenziert Neergard (2009: $20 \mathrm{ff}$.) Dienstleistungen der Daseinsvorsorge (Services of General Interest) entsprechend der Marktnähe in, erstens, Dienstleistungen von allgemeinem wirtschaftlichem Interesse (z. B. Netzinfrastrukturen aus den Bereichen Energie, Wasser/Trinkwasser, Telekommunikation, Transport) sowie, zweitens, nicht wirtschaftliche Dienstleistungen von allgemeinem Interesse (z. B. Dienstleistungen in Zusammenhang mit wohlfahrtsstaatlichen Sicherungssystemen, Bildung, Kinderbetreuung, Gesundheitsvorsorge, soziale 
Fürsorge, Kultur und (sozialen) Wohnungsbau). Wichtige Definitionskriterien für Leistungen der Daseinsvorsorge sind dabei ihr universeller, nicht exklusiver Zugang, die mittel- bis langfristig stabile und transparente Erbringung sowie die staatliche Kontrolle von Preisbildung und Qualität. Laut Neergard (2009: 20) sind davon marktbasierte Dienstleistungen (mit freier Angebots- und Preisgestaltung) sowie rein staatlich erbrachte Dienstleistungen (z. B. innere und äußere Sicherheit, Diplomatie) abzugrenzen.

Bund, Länder und Kommunen verfolgen in Deutschland das raumplanerische Ziel, für die Bürger in allen Regionen gleichwertige Lebensverhältnisse über Daseinsvorsorgeleistungen sicherzustellen (vgl. Kommunalpolitisches Forum Mecklenburg-Vorpommern 2011; Wollmann 2013; Brandt 2015). Einige dafür notwendige Aufgaben werden den Kommunen von Bund und Ländern übertragen und zählen zu den Pflichtaufgaben zur Erfüllung nach Weisung, da die Kommunen hier kaum Handlungsspielraum haben (z. B. Ausländerangelegenheiten, Ordnungsrecht, Katastrophenschutz, Unterhaltssicherung, Gesundheitsdienst). Bei Pflichtaufgaben ohne Weisung (Handlungsspielraum besteht in der Ausgestaltung; z. B. ÖPNV, Abfallwirtschaft, Sozialhilfe, Jugendhilfe, Brandschutz, Denkmalschutz) und bei freiwilligen Aufgaben (Handlungsspielraum besteht bezüglich der Erbringung; z. B. Sport und Erholung, Jugendund Kulturarbeit) haben die Kommunen die Möglichkeit, Angebote der Daseinsvorsorge gezielt als ,weiche Standortfaktoren " und Instrumente zur Steigerung der lokalen Lebensqualität einzusetzen. Gleichzeitig besteht insbesondere in finanzschwachen, vom demographischen Wandel stark betroffenen Kommunen bei diesen freiwilligen kommunalen Aufgaben die Gefahr der Reduzierung bis ersatzlosen Einstellung.

Gerade die bereits dünn besiedelten ländlichen Räume sind in Deutschland in besonderem Maße vom demographischen Wandel betroffen. Insbesondere in Ostdeutschland sind das Ausmaß von Schrumpfung und Abwanderung sowie die Dynamik der Alterung im europäischen Vergleich außerordentlich hoch (Wiest/Leibert/Johansson et al. 2011; Leibert 2012; Wolff/Leibert 2016). In den kommenden zehn bis 15 Jahren wird sich die demographische Situation allerdings auch in vielen Regionen Westdeutschlands deutlich verändern (Leibert/Lentz 2011). Dadurch stehen die vorhandenen Strukturen und Standards der Daseinsvorsorge dort unter erheblichem Anpassungsdruck. Bevölkerungsrückgang und Alterung sorgen im Bereich der sozialen Infrastrukturen für eine sich verändernde Nachfrage (Mai/ Swiaczny 2008: 12 ff.). Aber auch die Pro-Kopf-Kosten für die Daseinsvorsorge steigen und es kommt zunehmend zu Tragfähigkeitsproblemen (Winkler-Kühlken 2003). Gelingt es nicht, die Daseinsvorsorge neu zu gestalten, droht insbesondere dünn besiedelten Regionen außerhalb der Agglomerationen eine Abwärtsspirale aus Attraktivitätsverlusten durch den Rückbau von Angeboten, sinkender Lebensqualität und weiterer Abwanderung.

Zudem sieht sich die öffentliche Hand vielerorts einem Spardruck ausgesetzt, der dazu führt, dass insbesondere bestehende freiwillige Angebote der Daseinsvorsorge ausgedünnt oder eingestellt werden. Es steht zur Diskussion, inwieweit der Staat angesichts der hohen Kosten das bisherige Niveau der infrastrukturellen Versorgung noch aufrechterhalten kann und soll (Steinführer/Küpper/Tautz 2012; Hüther 2016). Damit verbunden ist eine Debatte um die Gleichwertigkeit der Lebensverhältnisse, die als eines der Grundprinzipien des deutschen Föderalismus gilt (Lehmbruch 2000; ARL 2008; ARL 2016). Hinterfragt wird hier, ob der Staat als alleiniger Leistungsträger von Daseinsvorsorgeleistungen zu verstehen sei oder nicht doch auch andere Akteure in die Verantwortung genommen werden sollten. Dabei wird neben der Wirtschaft auch die Zivilgesellschaft als Leistungserbringer diskutiert.

\section{2 Öffentliche Daseinsvorsorge und zivilgesellschaftliches Engagement}

Zivilgesellschaftliches Engagement wird neben der Wirtschaft und der öffentlichen Hand als eine der drei Säulen der Stadt- und Regionalentwicklung angesehen (Becker 2008). In Bezug auf die räumliche Planung und Entwicklung formuliert Becker (2008: 120) zwei Grundannahmen: Zivilgesellschaftliches Engagement muss, erstens, nicht zwangsläufig einen Gegenpol zum Staat darstellen und es kann, zweitens, finanziell und inhaltlich von der öffentlichen Hand beeinflusst (und damit nicht per se von Staat und Markt unabhängig) sein. Daraus ergibt sich am Ende die Frage nach der Positionierung der öffentlichen Hand gegenüber der Zivilgesellschaft: „Will man gesteuert Aufgaben teilen oder will man mündige Bürger/innen, die von sich aus ihre Meinung sagen?" (Becker 2008: 122).

Auch wenn diese Frage bisher in der Kommunalpraxis nicht klar beantwortet wird, wird zivilgesellschaftliches Engagement im Kontext der Stadt- und Regionalentwicklungspolitik als wichtiges Werkzeug bzw. als wichtiger Themenbereich verstanden (Becker 2015). So beschäftigen sich weite Teile in den Beschreibungen zur ,Nationalen Stadtentwicklungspolitik“ (BMVBS 2008) und zum Programm „Soziale Stadt“ (BMVBS 2012) mit zivilgesellschaftlichem Engagement. Zu diesen Programmen wird aber auch festgestellt, dass in Bezug auf Zivilgesellschaft meist die Bürgerbeteiligung in Planungsprozessen betont und das zivilgesellschaftliche Engagement einer Top-Down-Logik unterworfen werden (Becker 2008: 122; Gualini 2010: 3).

Davon ausgehend betrachtet Siebel (2010) das Verhältnis von Planung (als hoheitlicher Aufgabe) und Zivilgesellschaft. Für ein Erstarken zivilgesellschaftlicher Akteure in räumlichen Planungs- und Entwicklungsprozessen führt 
er verschiedene Gründe an. Einerseits sei die vermehrte Orientierung auf zivilgesellschaftliche Akteure einer neoliberalen Ideologie mit ihrer Privatisierungslogik und einer strukturellen Unterfinanzierung öffentlicher und kommunaler Akteure geschuldet (vgl. auch Brenner/Theodore 2002a; Brenner/Theodore 2002b). In diesem Zusammenhang soll zivilgesellschaftliches Engagement als neue Ressource für die Bereitstellung von Daseinsvorsorgeleistungen erschlossen werden. Andererseits seien neue Themen wie urbane Milieus, soziale Ungleichheit sowie Exklusions- und Marginalisierungsprozesse in der Stadtgesellschaft in den Fokus der Stadtentwicklung gerückt, bei denen die Instrumente der klassischen Angebotsplanung nicht mehr greifen (Siebel 2010).

Zivilgesellschaftliches Engagement wird auch im ländlichen Raum bzw. in dörflichen Kontexten betrachtet, wobei hervorgehoben wird, dass Engagement hier eher von Einzelpersonen als von Gruppen ausgeht und traditionelle Sport-, Kultur- und Faschingsvereine sowie die Freiwillige Feuerwehr eine besondere Bedeutung haben (Borstel 2010: 93). Im Gegensatz zu großstädtischen Gesellschaften ist die Zivilgesellschaft im dörflichen Kontext eher Ausdruck von konsensualen Meinungen und ermöglicht einen positiven Gemeinwesenbezug (Borstel 2010: 93 ff.). Die direkte Betroffenheit und ein hohes Verantwortungsbewusstsein im ländlichen Raum führten zu einer hohen Engagementbereitschaft. Allerdings wird ein Wandel von einem langfristigen, am Gemeinwesen orientierten hin zu einem stärker temporären und projektbezogenen Engagement beobachtet (vgl. Becker/Runkel 2010: $132 \mathrm{f}$.). Betont wird auch die Nähe der Zivilgesellschaft zu Politik und Verwaltung im ländlichen Raum (Becker/Runkel 2010: 139).

Zusammenfassend lässt sich festhalten, dass die Zivilgesellschaft von öffentlichen Akteuren, die mit der Erbringung von Daseinsvorsorgeleistungen betraut sind, traditionell als wichtiger Partner wahrgenommen wird und der Wunsch besteht, zivilgesellschaftliche Akteure bei der Gestaltung des gesellschaftlichen Miteinanders gezielt einzubinden. Aus kritischer Perspektive kann die mit diesem Wunsch zunehmend verbundene Substitutionslogik als Bestandteil einer neoliberalen Agenda interpretiert werden, durch die vormals durch öffentliche Akteure in Erwerbsarbeit erbrachte Dienstleistungen im Bereich der Daseinsvorsorge perspektivisch stärker durch freiwillige Zeitspenden von Bürgerinnen und Bürgern erbracht werden sollen. Dabei riskieren zivilgesellschaftliche Akteure, teilweise zu Erfüllungsgehilfen von Politik und Verwaltung degradiert zu werden. Aus einer positiven Sichtweise können jedoch auch die neuen Formen der gesellschaftlichen Teilhabe durch Bürgerinnen und Bürger in hoheitlichen Planungs- und Daseinsvorsorgeaufgaben betont werden, die neben einer stärkeren Selbstverantwortung auch eine selbstbestimmtere Gestaltung auf lokaler Ebene ermöglichen könnten.

\subsection{Die Substitutionslogik: Daseinsvorsorge durch zivilgesellschaftliches Engagement als Bewältigungsstrategie im demographischen Wandel}

Um die Folgen der als unvermeidbar angesehenen Anpassungen in peripheren Schrumpfungsregionen abzumildern, wird dabei an die Selbstverantwortung und Eigeninitiative der dort lebenden Bürgerinnen und Bürger appelliert (z. B. Aring 2013). In Zeiten, in denen ein allumfassender Wohlfahrtsstaat als nicht mehr finanzierbar angesehen wird, gilt die Aktivierung zivilgesellschaftlichen Engagements scheinbar als erfolgversprechende Strategie (Monheim 2005: 65). Mittels der Nutzung zivilgesellschaftlichen Engagements wird gehofft, in dünn besiedelten Räumen strukturelle Defizite ausgleichen und bestehende Angebote erhalten oder gar verbessern zu können (Mai/Swiaczny 2008; Steinführer 2015; BMFSFJ 2017).

Im Zuge der skizzierten demographischen Veränderungen in den vergangenen Jahrzehnten, verändert sich demnach das Verhältnis von Zivilgesellschaft und staatlichen Akteuren in Bezug auf die Erbringung von Daseinsvorsorgeleistungen. Dazu werden in der Kommunalpolitikforschung zwei Diskurse prominent geführt: Einerseits steht die Übertragung kommunaler Leistungen an private Unternehmer im Vordergrund von Privatisierungsansätzen (vgl. Häußermann/Läpple/Siebel 2008: 279 ff.). Andererseits und das ist hier für die Argumentation relevanter - wird die Kompensation entfallender staatlicher Leistungen durch zivilgesellschaftliches Engagement betont. Auf kommunaler Ebene spielt dabei das Konzept der Bürgerkommune eine wichtige Rolle, welches als Ergebnis eines veränderten Verhältnisses zwischen kommunaler Verwaltung und Politik auf der einen und Bürgerschaft auf der anderen Seite verstanden wird (Bogumil 2014). Wichtig ist hier, dass Bürgerinnen und Bürgern eine aktive Rolle in der kommunalpolitischen Zielfindung und Zielerreichung zukommt. So hat auch die Enquete-Kommission ,Zukunft des bürgerschaftlichen Engagements" des Deutschen Bundestages in ihren Handlungsempfehlungen die Notwendigkeit einer Förderung, Qualifizierung und Verstetigung zivilgesellschaftlichen Engagements zur kooperativen und auf Eigeninitiative basierenden Erbringung von Leistungen in den Kommunen betont (Deutscher Bundestag 2002: 19 ff.). Im Rahmen des „Siebten Berichts zur Lage der älteren Generation in der Bundesrepublik Deutschland" spricht die Bundesregierung von einer begrüßenswerten Auseinandersetzung mit dem Ordnungsprinzip der Subsidiarität und verweist auf die Aufgabe staatlicher Akteure, Möglichkeiten zu schaffen, um öffentliche Daseinsvorsorge durch die „eigenverantwortliche Sorge und Mitverantwortung von Bürgerinnen und Bürgern“ zu ergänzen (Deutscher Bundestag 2016: VIII).

Bereits seit den späten 1990er-Jahren intensivierte sich der Diskurs über die Potenziale des zivilgesellschaftlichen 
Engagements in der Wissenschaft ebenso wie in der Politik (Haus 2002). Gesellschaftliche Modernisierungsprozesse, wie die Auflösung tradierter kultureller, ökonomischer oder staatlicher Bindungen, wurden in Verbindung gebracht mit „Chancen [...] für die Entwicklung neuer oder für den Wandel alter zivilgesellschaftlicher Gemeinschaftsformen“ auf kommunaler Ebene (Schubert 2002: 18). Daher wird auch bisher weniger beteiligten Gruppen, wie jungen Frauen oder Migranten, eine positive Rolle bei der Gestaltung lokaler Gemeinschaft im demographischen Wandel zugeschrieben, die von Seiten der Politik entsprechend angeleitet und motiviert werden müsste (Nadler 2012; Wiest/ Leibert 2013).

In der Raumplanung wird mehr und mehr akzeptiert, dass die Gleichwertigkeit der Lebensverhältnisse in der derzeitigen Form nicht mehr finanzierbar sei und die freiwilligen Aufgaben der Daseinsvorsorge kaum noch flächendeckend erfüllt werden können (ARL 2016). Damit einhergehend wird diskutiert, ob sich staatlich erbrachte Daseinsvorsorge aus der Fläche zurückziehen solle und anstelle der alten Förderpolitik nach dem Gießkannenprinzip eine wachstumsorientierte und räumlich differenzierte Alternative zu implementieren sei, die auf Wachstumsinseln konzentriert bliebe (Eich-Born 2009: 228), während für periphere Räume lediglich Auffang- und Stabilisierungsstrategien vorgesehen wären (Aring 2013). Um die Folgen des staatlichen Rückzugs abzumildern, sollen lokale Politik und Planung an der Steuerungs- und Gestaltungsphilosophie des „Ermöglichens“ (ARL 2008: 7) im Sinne einer eigenständigen und selbstverantwortlichen Regionalpolitik mit weitem Handlungsspielraum der kommunalen Ebene ausgerichtet werden. Ein zentraler und viel diskutierter Aspekt des „Ermöglichens“ ist dabei die Übernahme von Bereichen bisher staatlich organisierter Daseinsvorsorge durch zivilgesellschaftliche Akteure im Rahmen deren zivilgesellschaftlichen Engagements - also kurz gesagt: eine Substitutionslogik.

\section{Discussing the Elephant in the Room}

\subsection{Dilemmata der Substitutionslogik}

Es dürfte nachvollziehbar sein, dass die Konzepte wie „Selbstverantwortung“ und „Ermöglichen“ im Rahmen der Substitutionslogik keine Musterpläne darstellen, die in allen Teilräumen gleich gut funktionieren (vgl. Kapitel 2.2). Jedoch wird über die Grenzen der Substitutionslogik ähnlich der Figur eines Elephant in the Room, also eines offensichtlichen Problems, das keiner anspricht - in der diskursiven Verbindung von demographischem Wandel, Daseinsvorsorge und zivilgesellschaftlichem Engagement kaum offen gesprochen. Ich möchte im Folgenden diesem
Elefanten auf seine vier Beine verhelfen und anhand verschiedener Dilemmata aufzeigen, welche Probleme sich bei der Umsetzung einer vereinfachten Substitutionslogik ergeben.

\subsubsection{Geographisches Dilemma}

Eisentraut (2009) betont die Verringerung des Ehrenamtspotenzials durch die schnell voranschreitende Alterung im ländlichen Raum und stellt fest, dass beispielsweise auBerfamiliäre Generationenbeziehungen in diesem Zusammenhang von Politik und Planung bisher ungenügend als Ressource wahrgenommen werden. Auch Liebmann (2010) verweist auf die aus Schrumpfungsbedingungen resultierenden Probleme für das zivilgesellschaftliche Engagement, die sich in Abhängigkeit von verschiedenen Faktoren wie lokaler sozialer Lage, Wohlstand, Vereinsmitgliedschaften, Bevölkerungs- und Familienstrukturen ergeben können. Explizit wird durch Liebmann auf mögliche negative Folgen der mit dem demographischen Wandel einhergehenden Lücken in sozialen Netzwerken hingewiesen, die antidemokratischen Kräften neue Möglichkeiten eröffnen, sich in der Gestaltung der Gesellschaft vor Ort einzubringen (Liebmann 2010: 82). Gleichzeitig wurde in Kapitel 3 beschrieben, dass sich der demographische Wandel in einzelnen Teilräumen Deutschlands unterschiedlich auswirkt. Der demographische Wandel führt also zu einem - regional differenziert - steigenden Bedarf nach zivilgesellschaftlichem Engagement, um Daseinsvorsorge in „Koproduktion“ mit staatlichen Akteuren zu erbringen (ARL 2016: 33); er bedingt gleichzeitig jedoch in einzelnen Regionen die Verringerung des Bevölkerungspotenzials für zivilgesellschaftliches Engagement. Durch die massive Ausdünnung sozialer Netzwerke fehlen gerade den ländlichen Regionen die Menschen, die das selbstverantwortliche Organisieren der Daseinsvorsorge bewerkstelligen könnten (Swiaczny 2010; Burdack/Kriszan/Nadler 2011). Selbst in peripheren Mittelzentren wird bereits auf diese negativen Auswirkungen der Abwanderung und Schrumpfung hingewiesen (Burdack 2010; Bernt/Liebmann 2013: 229 f.). Dies ist insbesondere vor dem Hintergrund bedeutsam, dass das Bevölkerungspotenzial für zivilgesellschaftliches Engagement aufgrund des demographischen Wandels in ländlich-peripheren Regionen besonders gering ist, wo gleichzeitig die Finanzierbarkeit und Tragfähigkeit der Daseinsvorsorge aufgrund der geringen Einwohnerdichte die größten Probleme bereiten (Mai/ Swiaczny 2008: 52). Auch wird in Studien darauf hingewiesen, dass gerade in Gemeinden, in denen sozial schwache und bildungsferne Bevölkerungsgruppen leben, solche Appelle an das zivilgesellschaftliche Engagement sehr voraussetzungsvoll sind (Klatt/Walter 2011). Insbesondere die sozialräumliche Selektivität in Fragen zivilgesellschaftlichen Engagements ist bereits mehrfach hervorgehoben worden 
(vgl. Mai/Swiaczny 2008: $31 \mathrm{ff}$.). In der Summe zeigt sich also das geographische Dilemma der Substitutionslogik: Zivilgesellschaftliches Engagement ist eigentlich dort am nötigsten, wo das Bevölkerungs- und damit das Engagementpotenzial bereits gering und tendenziell rückläufig sind.

\subsubsection{Politisches Dilemma}

Staatlichen Akteuren wird bisher eine große Gestaltungsmacht zugeschrieben (vgl. Levin-Keitel/Lelong/Thaler 2017). Mit dem Verlust finanzieller Handlungsspielräume geht bei staatlichen Akteuren auch häufig ein Machtverlust einher. So werden Kommunen bei zu hoher Verschuldung unter die Finanzaufsicht der Landesbehörden gestellt und können häufig auch ihre Aufgabengestaltung und Personalangelegenheiten nicht mehr autonom bestimmen. Dieser Verlust von durch finanzielle und personelle Ressourcen abgesicherter Macht wird dann häufig über das Durchsetzen symbolischer Macht gegenüber den Bürgerinnen und Bürgern kompensiert (vgl. Bourdieu 1992: $81 \mathrm{ff}$.). Anders ausgedrückt: Kommunale Akteure wollen zwar weiterhin Stadtentwicklung und Gemeinwesen definieren, aber sie haben nicht mehr die Ressourcen zur vollumfänglichen Umsetzung. Im Ergebnis entscheiden Akteure aus lokaler Politik und Verwaltung, welche Kernthemen mit den verbleibenden Ressourcen innerhalb der Verwaltung dem Machterhalt dienlich sind und welche Themen und Daseinsvorsorgebereiche in die Bürgerschaft, entlassen werden können und dort umgesetzt werden sollen. Hier findet also eine Top-Down-Logik der Themensetzung und Feldbeschreibung für, gewünschtes“ zivilgesellschaftliches Engagement statt. Dies widerspricht allerdings den Mechanismen zivilgesellschaftlichen Engagements, welches unter anderem durch die Berücksichtigung von Eigeninteressen der Engagierten motiviert wird. Soll also zivilgesellschaftliches Engagement stärker in die Daseinsvorsorge eingebunden werden, so treffen politische Interessen der Kommunen auf die Eigeninteressen der Engagierten und es muss zu einem Interessenausgleich kommen. Wie bereits in Kapitel 2 beschrieben, übt Zivilgesellschaft per Definition eigentlich keine politische Macht aus, da diese dem Zusammenwirken von Politik und öffentlicher Verwaltung vorbehalten ist. Ein grundsätzliches Problem besteht nun aber darin, dass für eine konsequente Umsetzung der Substitutionslogik staatliche Akteure Macht an zivilgesellschaftliche Akteure abgeben müssten. So sprechen Bogumil und Holtkamp (2002: 81 ff.) von der ,Selbstentmachtung der Kommunalvertretungen“. Im Umkehrschluss würde die partielle Entmachtung der staatlichen Akteure aus Politik und Verwaltung ein Empowerment zivilgesellschaftlicher Gruppen im strengen Wortsinn ergeben. Staatliche Akteure fürchten jedoch diesen Verlust von Macht und Deutungshoheit. Problematisch ist zudem, dass eine Übertragung von Macht und
Entscheidungshoheit auf zivilgesellschaftliche Gruppen im Kontext unseres Demokratieverständnisses ein legitimatorisches Problem mit sich bringen würde, da eben nicht mehr demokratisch gewählte Akteure bestimmen würden. Daneben wird in der Literatur zu Local Governance teilweise die Effizienz des Einbezugs zivilgesellschaftlicher Akteure kritisch betrachtet, da empirische Ergebnisse zeigen, dass Akteure aus Politik und Verwaltung zivilgesellschaftliche Partner als zu wenig engagiert erachten und Engagierte sich häufig im Kreis der hauptamtlichen Experten nicht ernst genommen fühlen (Geißel 2007). Schließlich wird auch ein zu hohes Maß an Regierungsintervention in Fragen der Aktivierung der Zivilgesellschaft kritisiert, da dies mit der Indienstnahme der Bürgerinnen und Bürger zum Zwecke des staatlichen Machterhalts einhergehen kann (Embacher 2011). Grundsätzlich besteht also das zweite Dilemma der Substitutionslogik darin, dass staatliche Akteure zivilgesellschaftliche Akteure als Partner für die Erbringung von hoheitlichen Aufgaben gewinnen wollen, für die Erstere eigentlich keine Macht und Deutungshoheit abgeben wollen und Letztere eigentlich keine demokratisch legitimierten Befugnisse haben.

\subsubsection{Motivationales Dilemma}

Per Definition ist zivilgesellschaftliches Engagement über eine freiwillige und unentgeltliche Arbeitszeitspende charakterisiert, die sowohl durch das Verfolgen von Eigeninteressen als auch im Hinblick auf Gemeinwohlorientierung motiviert ist. Zudem konnten die empirischen Ergebnisse zum zivilgesellschaftlichen Engagement in Deutschland zeigen, dass Engagement sich hin zu flexibleren Formen entwickelt, zunehmend die institutionellen Rahmen von Vereinen und Verbänden verlässt und temporäre, problembezogene Formen annimmt (vgl. Kapitel 2.2). Damit ist klar, dass mit einer stärkeren Einbindung von zivilgesellschaftlichen Akteuren in die Erbringung von vormals staatlichen Leistungen auch ein motivationales Dilemma in Bezug auf die Verbindlichkeit von Engagement einhergeht. So müssen in der Erbringung von Daseinsvorsorgeleistungen bestimmte Aufgaben per Gesetz abgedeckt werden und dafür ist eine gewisse Verbindlichkeit erforderlich. Letztlich aber zeichnet sich zivilgesellschaftliches Engagement ja dadurch aus, dass damit keine Aneignung von Arbeitsleistung durch die Wirtschaft oder den Staat einhergeht und Ehrenamt selbstbestimmt erfolgen sollte. Denn sonst wird die Substitutionslogik im Sinne der Kritik Siebels (2010) oder Mayers (2005) tatsächlich Bestandteil einer neoliberalen Agenda. In der Konsequenz entziehen sich zivilgesellschaftliche Partner der verbindlichen Arbeitsleistungseinforderung durch staatliche Akteure. Hier sei wieder an die eingangs zitierte Frage von Becker (2008) erinnert, ob man Aufgaben auf Bürgerinnen und Bürger verteilen will 
oder deren eigenen Gestaltungswillen respektiert? Die Substitutionslogik berücksichtigt bisher kaum die Bedürfnisse der Bürgerinnen und Bürger, die als Engagierte Lebenszeit in die Gestaltung des lokalen Zusammenlebens spenden sollen. Entsprechend der in Kapitel 2 skizzierten Definition zeichnet sich zivilgesellschaftliches Engagement gerade dadurch aus, dass hierbei Bürgerinnen und Bürger selbstregiert und demokratisch ein eigenes Projekt verfolgen können. Die derzeit praktizierte Planungskultur jedoch nimmt diesen Punkt nicht ernst. Selbstbestimmtes Mitgestalten ist darin nicht vorgesehen, da Verwaltung und Politik sich bisher noch immer als die einzigen legitimen Gestalterinnen verstehen, die Recht und Ordnung durchsetzen. Bürgerinnen und Bürger haben darin lediglich die Rolle des Assistenten bei der Durchsetzung. Damit stehen staatlichen Akteuren ihre notwendigerweise langfristig verfügbaren Ansprechpartner in der Zivilgesellschaft nicht in ausreichend motivierter Weise zur Verfügung und die Substitutionslogik sieht sich mit dem Dilemma konfrontiert, dass Bürgerinnen und Bürger häufig im Ehrenamt Leistungen erbringen sollen, die teilweise gar nicht ihren Eigeninteressen entsprechen und zu deren Erbringung sie sich zunehmend weniger binden lassen wollen.

\subsection{Neue Verbindungslinien zwischen demographischem Wandel, Daseinsvorsorge und zivilgesellschaftlichem Engagement}

Angesichts der zuvor beschriebenen Dilemmata möchte ich an dieser Stelle den Versuch unternehmen, die Verbindungslinien zwischen demographischem Wandel, Daseinsvorsorge und zivilgesellschaftlichem Engagement realistischer zu zeichnen. Dem geographischen Dilemma müssen zwei zentrale Beobachtungen hinzugefügt werden. Zum einen ist das bestehende Ehrenamtspotenzial in seiner regionalen und sozialen Differenziertheit noch nicht ausreichend erforscht und kann entsprechend noch nicht effizient mobilisiert werden. Neben den aus dem demographischen Wandel resultierenden Nachteilen ländlicher Regionen können diese sich beispielsweise auch die Spezifika ihrer Ehrenamtsstrukturen - starke Konsensorientierung, große traditionelle Nähe zwischen Politik und Zivilgesellschaft (vgl. Kapitel 2) zunutze machen. Hier besteht also ein mögliches Mobilisierungspotenzial für ländliche Räume. Zweitens ist zu konstatieren, dass - auch bei effizienterer Nutzung des bestehenden Ehrenamtspotenzials - durch die mit dem demographischen Wandel einhergehenden bevölkerungs- und siedlungsstrukturellen Ausdifferenzierungen gerade schrumpfende und schnell alternde Teilräume Deutschlands viel zu wenig Potenzial für eine Umsetzung der Substitutionslogik aufweisen. Gerade dort können staatliche Akteure keine Daseinsvorsorge in Selbstverantwortung erwarten und es ist zu diskutieren, ob ein raumdifferenzierender Ansatz in der
Finanzierung von Daseinsvorsorge sinnvoller ist, der anhand des Ehrenamtspotenzials verschiedene Substitutionsstufen unterscheidet. So könnten Substitutionslogiken mit gezielten Unterstützungsprogrammen gerade in den Regionen weniger intensiv umgesetzt werden, wo das Ehrenamtspotenzial geringer ist. Damit würde sich auch artikulieren, dass am Grundprinzip der Gleichwertigkeit der Lebensverhältnisse festgehalten wird.

In Bezug auf das politische Dilemma müssen Vertreterinnen und Vertreter staatlicher Organisationen ihr Selbstverständnis angesichts des durch die Verringerung personeller und finanzieller Handlungsspielräume ohnehin laufenden Machtverlustes im Bereich der Gestaltung und des Ausfüllens von Daseinsvorsorge - insbesondere in den freiwilligen Bereichen - verändern (vgl. Klemme 2010). Die bestehenden, eher asymmetrischen Relationen zwischen Politik und Verwaltung auf der einen Seite und zivilgesellschaftlichen Akteuren auf der anderen Seite müssen durch neue gleichberechtigtere Beziehungen ersetzt werden, in denen ehrenamtlich Engagierte als Partner auf Augenhöhe und ,Experten des Alltags' mit ihren Fähigkeiten und ihrem Wissen anerkannt und wertgeschätzt sowie in strategische Entscheidungsprozesse einbezogen werden. Dies ist grundlegende Bedingung für eine Koproduktion von Daseinsvorsorge, in die Bürgerinnen und Bürger bereitwillig Zeitspenden geben würden. Dafür sind Ansätze aus dem Bereich der Planungskulturforschung aufschlussreich, die einen Einblick in die Prozesse der Veränderung hinsichtlich des kollektiven Selbstverständnisses von Akteuren aus Politik und Verwaltung gewähren (vgl. Reimer 2016) bzw. einen analytischen Zugang zu deren Umgang mit Macht ermöglichen (LevinKeitel/Lelong/Thaler 2017). Ein möglicher Weg, diese neuen Rollenverständnisse in der Praxis zu testen, bestünde in der Schaffung von Modellregionen, in denen Bürger stärker in die Gestaltung der Substitution und Definition von Standards für die Daseinsvorsorge eingebunden werden.

Bezüglich des motivationalen Dilemmas müssen Anerkennungskulturen für zivilgesellschaftliches Engagement gestärkt werden. So sind die Eigeninteressen der zivilgesellschaftlich Engagierten stärker zu respektieren und von Seiten der Politik und Verwaltung zu berücksichtigen. Dafür ist jedoch ein viel grundlegenderes Verständnis dieser Eigeninteressen zu erarbeiten. Bisher ist dieses mangelhaft beforscht. Wenn jedoch gleichzeitig eine fortschreitende Individualisierung konstatiert wird, durch die Ehrenamt kurzfristiger, kleinteiliger und flexibler vonstattengeht, dann ist eine nach sozialen Gruppen und Milieus differenzierte Auswertung von Motiven und Bedürfnissen umso wichtiger. Sobald die Eigeninteressen ergründet sind, gilt es, aus den individuellen Motiven wiederum kollektive zivilgesellschaftliche Interessen $\mathrm{zu}$ abstrahieren. Denn nur wenn einzelne Bürgerinnen und Bürger ihre persönlichen Interessen mit anderen Bürgern teilen, kann es dauerhaft 
gelingen, zivilgesellschaftliche Gruppen zu bilden, die auf ein geteiltes Ziel hinarbeiten, solidarisch und am Gemeinwohl orientiert handeln. Hierfür sind Ansätze aus der Sozialpsychologie hilfreich. Der Ansatz des Opinion-Based Grouping ermöglicht beispielsweise, über kollektiv geteilte Meinungen und Unzufriedenheiten Gruppenidentitäten zu bilden, die wiederum Basis für kollektives Handeln sind (vgl. McGarty/Bliuc/Thomas et al. 2009). Diese Erkenntnisse aus der Sozialpsychologie könnten genutzt werden, um zivilgesellschaftliches Engagement zusätzlich zur verbesserten Anerkennungskultur zu motivieren. Gleichzeitig ist jedoch auch kritisch zu hinterfragen, welche Daseinsvorsorgebereiche welche Verbindlichkeit benötigen, und entsprechend muss differenziert werden, welche Teile der Daseinsvorsorge überhaupt in das Ehrenamt übertragbar sind.

\section{Schlussfolgerungen}

Insgesamt zeichnet sich derzeit in Deutschland eine Situation $\mathrm{ab}$, in der Kommunen zunehmend unter finanziellen Sparzwängen leiden und freiwillige Aufgaben der Daseinsvorsorge nicht mehr in Eigenleistung erbringen können. Darüber sind sowohl die Privatwirtschaft als auch die Zivilgesellschaft als mögliche Partner in den Blick gerückt. In wirtschaftsschwachen Regionen wird die Übernahme von Aufgabenerbringung in der Daseinsvorsorge durch die Privatwirtschaft kaum realistische Chancen haben. In der Konsequenz hoffen Vertreterinnen und Vertreter aus Politik und Verwaltung hier stärker auf zivilgesellschaftliches Engagement.

Angesichts der skizzierten Dilemmata - geographisch, politisch, motivational - stellt sich die derzeitige argumentative Verknüpfung von demographischem Wandel, zivilgesellschaftlichem Engagement und (der Umgestaltung von) Daseinsvorsorge zu einer Substitutionslogik in der deutschen Stadt- und Regionalentwicklung als Elephant in the Room dar. Über die offensichtlichen Probleme - unter anderem, dass bestimmte Teilgruppen der Gesellschaft unterproportional engagiert sind, dass Engagement genau in den Regionen gering ist, die es am stärksten bräuchten, dass Engagement sich hin zu flexibleren Formen mit verringerter Verantwortungsübernahme entwickelt, wobei Daseinsvorsorge eigentlich stabile Strukturen bräuchte - wird kaum offen diskutiert. Im Hinblick auf die zentrale Fragestellung dieses Beitrags kann geschlussfolgert werden, dass eine Kompensierung vormals staatlich erbrachter Daseinsvorsorgeleistungen durch zivilgesellschaftliches Engagement in der derzeitigen Konzeption keine ausreichend tragfähige Lösung zur Abmilderung negativer Auswirkungen des demographischen Wandels darstellt. Mittlerweile scheinen sich auch dahingehende Erwartungen seitens der Bundesregie- rung zu relativieren. So betont das Bundesministerium für Familie, Senioren, Frauen und Jugend (BMFSFJ 2017: 151) die Notwendigkeit der Achtsamkeit, dass „das subsidiäre Denken nicht als substitutives instrumentalisiert wird, der Staat sich von Aufgaben befreit, die er einst übernommen hat".

Aus der Dekonstruktion der gängigen Verbindungslinien zwischen demographischem Wandel, Daseinsvorsorge und zivilgesellschaftlichem Engagement wurden hier jedoch Ideen für eine Überarbeitung dieser Verbindungslinien skizziert, die weiterführender offener Diskussion und Forschungsarbeit bedürfen. So ist die Beforschung sozialgruppenspezifischer Motive und Hemmnisse für zivilgesellschaftliches Engagement notwendig. Des Weiteren fehlen Erkenntnisse über Aktivierungsmöglichkeiten bezüglich bisher ungenutzten Engagementpotenzials in der Bevölkerung. Es wird zwar teilweise auf ein zunehmendes Engagementpotenzial verwiesen, jedoch bedeutet dies nicht, dass sich das Potenzial in konkretem Handeln niederschlägt. Hierzu sind weitere Forschungen zu funktionierenden Aktivierungsmechanismen notwendig. Eine mögliche Stoßrichtung stellen dabei ermöglichende Rahmenbedingungen und die Beratung und Begleitung von Engagierten dar. In diesem Sinne empfiehlt beispielsweise Sittler (2015: 9), dass Engagementförderung haushaltsrechtlich als kommunale Pflichtaufgabe zu definieren sei.

Es zeigt sich in der bundesdeutschen Debatte und in den Forschungsergebnissen zum Thema zivilgesellschaftliches Engagement (ARL 2016; Romeu Gordo/Vogel 2017; Simonson/Vogel 2017), dass dieses mit einer adäquaten Anerkennungskultur unterstützt werden sollte. Offen ist hier jedoch, wie eine solche Anerkennungskultur aussehen kann. Hinsichtlich der Anerkennungskultur bemerken Bischoff, Hagedorn und Roscher (2015: 28), dass es neben Aufwandsentschädigungen durchaus andere Möglichkeiten wie gemeinsame Unternehmungen, Fortbildungen und Versicherungen für Engagierte gibt, aber ,die alltägliche Wertschätzung der konkreten Arbeit der Freiwilligen und das ,Dankeschön dazwischen “ werden von den [Engagierten] als wichtiger eingestuft als formale institutionalisierte Anerkennung". Dennoch fehlen bisher vertiefende Forschungen zu den Perspektiven der Engagierten. Es gilt zu hinterfragen, welche Art von Anerkennung aus der Sicht der Engagierten als angemessen wahrgenommen wird.

Bisherige Forschungen zeigen thesenhaft, dass es durch die Aufgabenübertragung aus der Kommune (Politik und Verwaltung) an zivilgesellschaftliche Träger zu einer Neuaushandlung von Beziehungen und Positionen zwischen beiden gesellschaftlichen Teilbereichen kommen wird. Empirisch wenig fundiert ist jedoch, wie dieser Aushandlungsprozess von Vertreterinnen und Vertretern beider Seiten wahrgenommen und interpretiert wird. Diese Erkenntnisse sind jedoch essenziell, um erfolgreiche Modelle der Über- 
tragung von Aufgaben entwickeln zu können. Mit dem bisherigen Wissensstand und der daraus abgeleiteten Substitutionslogik können seitens der Politik bestehende, aber überhöhte Hoffnungen und Erwartungen an zivilgesellschaftliches Engagement bisher fast nur enttäuscht werden. Zum Anstoß einer offenen Debatte über die realistischen Möglichkeiten der Substitution von staatlich erbrachter Daseinsvorsorge durch zivilgesellschaftliches Engagement ist zuallererst eine konsequente Hinwendung zu einem passenderen Engagementverständnis notwendig, bei dem die Eigeninteressen der Bürgerinnen und Bürger analysiert und berücksichtigt werden. Für ein Verständnis dieser Eigeninteressen ist weitere empirische Grundlagenforschung durchzuführen, die sich mit den Motiven und Bedürfnissen der Bürgerinnen und Bürger und ihrer Selbstpositionierung gegenüber staatlichen Akteuren auseinandersetzt. Dabei sind auch die Dimensionen der Emotionalität, Kreativität, Intersubjektivität und Sozialethik stringenter in die Analyse von zivilgesellschaftlichem Engagement einzubinden (vgl. Hollstein 2015).

Dieser Beitrag verfolgte das Ziel, die bestehende Substitutionslogik auf ihre innere Konstitution hin zu prüfen. Es wurden zentrale Probleme aufgezeigt und Verbesserungen angeregt. Was der Beitrag allerdings nicht leistete, war ein grundsätzliches Hinterfragen der Substitutionslogik auf übergeordneter Ebene. Doch auch dies ist aus sozialwissenschaftlicher Sicht ein möglicher und wichtiger Weg für zukünftige Forschung. Gerade hinsichtlich der Erkenntnisse der Peripherisierungsforschung (Ehrlich/ Kriszan/Lang 2012; Perrons 2012; Lang 2015; PoSCOPP 2015) lassen sich Rückzugsstrategien von Politik und Verwaltung vor dem Hintergrund neoliberaler Politiken kritisch reflektieren. Lang (2015) versteht Peripherisierung als multidimensionalen Prozess (sozial, politisch, wirtschaftlich), der sich auf verschiedenen räumlichen Maßstabsebenen abspielt und dialektisch mit Prozessen der Zentralisierung einhergeht. Aus dem Zusammenspiel von Peripherisierung und Zentralisierung ergeben sich sozialräumliche Polarisierungsprozesse, welche wiederum in Verbindung mit Diskursen über Räume stehen, woraus im Ergebnis einzelne Regionen symbolisch aufgewertet werden und andere Regionen eine Abwertung erfahren (vgl. auch Miggelbrink/ Meyer 2015). Gleichzeitig sind peripherisierte Regionen und Orte nicht als handlungsunfähig anzusehen und Prozesse der Peripherisierung und Polarisierung können durch das Handeln verschiedener Akteure durchaus auch umgekehrt werden. Lang (2015: 176) formuliert: „All sorts of actors have their (implicit and explicit) shares in these processes, hence peripheralised regions cannot be seen as victims of some overarching processes beyond their control without any agency to them. Neither peripheries nor centres can be seen as static concepts with naturally given features and boundaries. Peripheralisation and centralisation are dyna- mic processes which can be reversed, rejected or redirected in the long run."

Damit eröffnen sich im Hinblick auf die diskursiven Verbindungslinien zwischen demographischem Wandel, Daseinsvorsorge und zivilgesellschaftlichem Engagement neue Forschungsfragen nach der Rolle verschiedener Akteursgruppen im Diskurs über genau diese Verbindungslinien. Es ist also zukünftig auch näher zu untersuchen, mit welchen Motiven verschiedene Akteure Positionen für oder gegen die bestehende Substitutionslogik einnehmen.

\section{Literatur}

Adloff, F. (2005): Zivilgesellschaft. Theorie und politische Praxis. Frankfurt am Main.

Aring, J. (2013): Inverse Frontiers - Selbstverantwortungsräume. In: Faber, K.; Oswalt, P. (Hrsg.): Raumpioniere in ländlichen Regionen - Neue Wege der Daseinsvorsorge. Leipzig, 42-56. = Edition Bauhaus 35.

ARL - Akademie für Raumforschung und Landesplanung (2008): Politik für periphere, ländliche Räume: Für eine eigenständige und selbstverantwortliche Regionalentwicklung. Hannover. = Positionspapier aus der ARL 77.

ARL - Akademie für Raumforschung und Landesplanung (2016): Daseinsvorsorge und gleichwertige Lebensverhältnisse neu denken - Perspektiven und Handlungsfelder. Hannover. = Positionspapier aus der ARL 108.

BAGFA - Bundesarbeitsgemeinschaft der Freiwilligenagenturen (2014): Monetarisierung - kein Weg zur Förderung des bürgerschaftlichen Engagements. Berlin.

Becker, E. (2008): Alle reden über Zivilgesellschaft. Differenzierung eines gesellschaftlichen Phänomens in der Stadtentwicklung. In: RaumPlanung 138/139, 119-123.

Becker, E. (2015): Zivilgesellschaft, Protestbewegung und Stadtentwicklung: (k)ein neues Thema? In: Othengrafen, F.; Sondermann, M. (Hrsg.): Städtische Planungskulturen im Spiegel von Konflikten, Protesten und Initiativen. Berlin, 59-83. = Planungsrundschau 23.

Becker, E.; Runkel, C. (2010): Zivilgesellschaft in räumlichen Arenen. In: Becker, E.; Gualini, E.; Runkel, C.; Graf Strachwitz, R. (Hrsg.): Stadtentwicklung, Zivilgesellschaft und bürgerschaftliches Engagement. Stuttgart, 121-203. = Maecenata-Schriften 6.

Bernt, M.; Liebmann, H. (2013): Zwischenbilanz: Ergebnisse und Schlussfolgerungen des Forschungsprojektes. In: Bernt, M.; Liebmann, H. (Hrsg.): Peripherisierung, Stigmatisierung, Abhängigkeit? Deutsche Mittelstädte und ihr Umgang mit Peripherisierungsprozessen. Wiesbaden, 218-231. doi: 10.1007/978-3531-19130-0_11

Bischoff, A.; Hagedorn, S.; Roscher, R. (2015): StiftungsStudie: Freiwilliges Engagement in Stiftungen. Berlin.

BMFSFJ - Bundesministerium für Familie, Senioren, Frauen und Jugend (2017): Zweiter Bericht über die Entwicklung des bürgerschaftlichen Engagements in der Bundesrepublik Deutschland. Schwerpunktthema: „Demografischer Wandel und bürgerschaftliches Engagement: Der Beitrag des Engagements zur lokalen Entwicklung". Berlin.

BMVBS - Bundesministerium für Verkehr, Bau und Stadtentwicklung (2008): Nationale Stadtentwicklungspolitik. Eine Initiative zur Stärkung der Zukunftsfähigkeit deutscher Städte. Berlin.

BMVBS - Bundesministerium für Verkehr, Bau und Stadtentwicklung (Hrsg.) (2012): Programme des Bundes für die nachhaltige Stadtentwicklung und Soziale Stadt. Berlin. = BMVBS-Online-Publikation 3/2012. 
BMVBS - Bundesministerium für Verkehr, Bau und Stadtentwicklung (Hrsg.) (2013): Daseinsvorsorge im europäischen Vergleich Problemwahrnehmung, Lösungsstrategien, Maßnahmen. Berlin. $=$ BMVBS-Online-Publikation 04/2013.

Bogumil, J. (2014): Zivilgesellschaftliche Modelle: Bürgerkommune und Kommunalverwaltung. In: König, K.; Kropp, S.; Kuhlmann, S.; Reichard, C.; Sommermann, K.-P.; Ziekow, J. (Hrsg.): Grundmuster der Verwaltungskultur. Interdisziplinäre Diskurse über kulturelle Grundformen der öffentlichen Verwaltung. BadenBaden, 415-432. doi: 10.5771/9783845255064_415

Bogumil, J.; Holtkamp, L. (2002): Liberalisierung und Privatisierung kommunaler Aufgaben - Auswirkungen auf das kommunale Entscheidungssystem. In: Libbe, J.; Tomerius, S.; Trapp, J. H. (Hrsg.): Liberalisierung und Privatisierung kommunaler Aufgabenerfüllung. Soziale und umweltpolitische Perspektiven im Zeichen des Wettbewerbs. Berlin, 71-91. = Difu-Beiträge zur Stadtforschung 37 .

Borstel, D. (2010): Zivilgesellschaft in dörflichen Kontexten. Eine ostdeutsche Perspektive. In: Becker, E.; Gualini, E.; Runkel, C.; Graf Strachwitz, R. (Hrsg.): Stadtentwicklung, Zivilgesellschaft und bürgerschaftliches Engagement. Stuttgart, 85-98. = MaecenataSchriften 6.

Bourdieu, P. (1992): Die verborgenen Mechanismen der Macht. Hamburg. $=$ Schriften zu Politik \& Kultur 1.

Brandt, E. (2015): Gleichwertige Lebensverhältnisse - verfassungsrechtliche Grundlagen. In: Informationen zur Raumentwicklung $1,1-9$.

Brenner, N.; Theodore, N. (2002a): Cities and the Geographies of "Actually Existing Neoliberalism”. In: Antipode 34, 3, 349-379. doi: $10.1111 / 1467-8330.00246$

Brenner, N.; Theodore, N. (2002b): Preface: From the 'New Localism' to the Spaces of Neoliberalism. In: Brenner, N.; Theodore, N. (Hrsg.): Spaces of Neoliberalism. Urban Restructuring in North America and Western Europe. Oxford/Malden, v-xi.

Burdack, J. (2010): Small town development and social capital in Eastern Germany: the cases Colditz and Leisnig. In: Borsig, A.; Burdack, J.; Knappe, E. (Hrsg.): Small towns in Eastern Europe: local networks and urban development. Leipzig, 26-45. = Beiträge zur Regionalen Geographie 64.

Burdack, J.; Kriszan, M.; Nadler, R. (2011): Die Bedeutung von Netzwerken für Prozesse regionalen Lernens in ländlich geprägten $\mathrm{Ge}-$ bieten Ostsachsens. In: Hron, R.; Keßler, I.; Kost, K.; Röhrig, R. (Hrsg.): Netzwerke der Mitbestimmung - Beteiligung jenseits der Alltagsnorm. Das Betriebs- und Personalrätenetzwerk „Canaletto" und die Notwendigkeit und Umsetzung mitbestimmter Netzwerke. Marburg, 105-133.

Deutscher Bundestag (2002): Bericht der Enquete-Kommission „Zukunft des Bürgerschaftlichen Engagements“. Bürgerschaftliches Engagement: auf dem Weg in eine zukunftsfähige Bürgergesellschaft. Berlin. = Drucksache 14/8900 vom 3. Juni 2002.

Deutscher Bundestag (2016): Siebter Bericht zur Lage der älteren Generation in der Bundesrepublik Deutschland. Sorge und Mitverantwortung in der Kommune - Aufbau und Sicherung zukunftsfähiger Gemeinschaften. Berlin. Drucksache 18/10210 vom 2. November 2016.

DGB - Deutscher Gewerkschaftsbund (2016): Mobilität in der Arbeitswelt: Immer mehr Pendler, immer größere Distanzen. Berlin. = Arbeitsmarkt aktuell 2/2016.

Ehrlich, K.; Kriszan, A.; Lang, T. (2012): Urban Development in Central and Eastern Europe - Between Peripheralization and Centralization? In: disP - The Planning Review 48, 2, 77-92. doi: $10.1080 / 02513625.2012 .721611$

Eich-Born, M. (2009): Räumlich differenzierte Entwicklungs- und Förderstrategien für Nordostdeutschland 2006+: Ein Fazit. In: Eich-Born, M. (Hrsg.): Räumlich differenzierte Entwicklungsund Förderstrategien für Nordostdeutschland. Hannover, 221229. = Arbeitsmaterial der ARL 345.
Eisentraut, R. (2009): Ausblick. In: Eisentraut, R. (Hrsg.): Alternde Räume: Soziales Kapital und ländlicher Raum Sachsen-Anhalts. Entwicklung von Lebensqualität im ländlichen Raum SachsenAnhalts. Ergebnisse eines Studentischen Lehrforschungsprojektes. Halle, 38-39. = Der Hallesche Graureiher: Forschungsberichte des Instituts für Soziologie 2009-2.

Embacher, S. (2011): Wenig Staat, zu viel Regierung: Irrungen und Wirrungen aktueller Engagementpolitik. In: Forschungsjournal Soziale Bewegungen 24, 3, 82-89. doi: 10.1515/fjsb-2011-0309

Enste, D. H.; Neumann, M.; Schare, T. (2012): Erster Engagementbericht 2012. Für eine Kultur der Mitverantwortung. Berlin.

Geißel, B. (2007): Zur (Un-)Möglichkeit von Local Governance mit Zivilgesellschaft: Konzepte und empirische Befunde. In: Schwalb, L.; Walk, H. (Hrsg.): Local Governance - mehr Transparenz und Bürgernähe? Wiesbaden, 23-38. = Bürgergesellschaft und Demokratie 24. doi: 10.1007/978-3-531-90571-6_2

Gualini, E. (2010): Zivilgesellschaftliches Handeln und bürgerschaftliches Engagement aus stadtentwicklungspolitischer Perspektive. Kritische Überlegungen zur Thematik. In: Becker, E.; Gualini, E.; Runkel, C.; Graf Strachwitz, R. (Hrsg.): Stadtentwicklung, Zivilgesellschaft und bürgerschaftliches Engagement. Stuttgart, 3-22. $=$ Maecenata-Schriften 6 .

Haus, M. (2002): Einleitung: Lokale Politikforschung als Frage nach Bürgergesellschaft und sozialem Kapital. In: Haus, M. (Hrsg.): Bürgergesellschaft, soziales Kapital und lokale Politik. Theoretische Analysen und empirische Befunde. Opladen, 9-29. = Stadtforschung aktuell 86. doi: 10.1007/978-3-663-09455-5_2

Häußermann, H.; Läpple, D.; Siebel, W. (2008): Stadtpolitik. Frankfurt am Main.

Heinrich, G. (2005): Rechtsextremismus in Mecklenburg-Vorpommern - Ein Überblick. In: Heinrich, G. (Hrsg.): Beiträge zu Fragen von Zivilgesellschaft und Rechtsextremismus. Rostock, 23-39. = Rostocker Informationen zu Politik und Verwaltung 25.

Hollstein, B. (2015): Ehrenamt verstehen. Eine handlungstheoretische Analyse. Frankfurt am Main/New York.

Hüther, M. (2016): Demografiepolitik zur gesellschaftlichen Transformation: Möglichkeiten und Grenzen. In: Mayer, T. (Hrsg.): Die transformative Macht der Demografie. Wiesbaden, 459-475. doi: 10.1007/978-3-658-13166-1_28

Kern, K. (2004): Sozialkapital, Netzwerke und Demokratie. In: Klein, A.; Kern, K.; Geißel, B.; Berger, M. (Hrsg.): Zivilgesellschaft und Sozialkapital. Herausforderungen politischer und sozialer Integration. Wiesbaden, 109-129. = Bürgergesellschaft und Demokratie 14. doi: 10.1007/978-3-322-80963-6_8

Klatt, J.; Walter, F. (2011): Entbehrliche der Bürgergesellschaft? Sozial Benachteiligte und Engagement. Bielefeld.

Klemme, M. (2010): Stadtentwicklung ohne Wachstum. Zur Praxis kommunaler Siedlungsflächenentwicklung. Empirische Befunde und Folgerungen zu Steuerungsverständnissen und -formen öffentlicher Akteure. Saarbrücken.

Kocks, M. (2005): Demographischer Wandel in dünn besiedelten, strukturschwachen Räumen. Diskussionsbericht. In: Strubelt, W.; Zimmermann, H. (Hrsg.): Demographischer Wandel im Raum: Was tun wir? Gemeinsamer Kongress 2004 von ARL und BBR. Hannover, 74-78. $=$ Forschungs- und Sitzungsberichte der ARL 225.

Kommunalpolitisches Forum Mecklenburg-Vorpommern (2011): Was verbirgt sich hinter den so genannten Pflicht- und Freiwilligen Aufgaben? Schwerin. http://www.kf-mv.de/Themen/ Kommunalfinanzen/thema01_002.htm (22.08.2017).

Lang, T. (2015): Socio-economic and political responses to regional polarisation and socio-spatial peripheralisation in Central and Eastern Europe: a research agenda. In: Hungarian Geographical Bulletin 64, 3, 171-185. doi: 10.15201/hungeobull.64.3.2

Lehmbruch, G. (2000): Parteienwettbewerb im Bundesstaat. Regelsysteme und Spannungslagen im politischen System der Bundesre- 
publik Deutschland. Wiesbaden. doi: 10.1007/978-3-322-808523

Leibert, T. (2012): „Männerproletariat“ - schiffbrüchig im „Ozean von Armut und Demenz"? Die Geschlechterproportionen als Indikator für die sozioökonomische Marginalisierung ländlicher Räume Ostdeutschlands. In: Statistischer Quartalsbericht der Stadt Leipzig IV/2011 [01/12], 20-28.

Leibert, T.; Lentz, S. (2011): Die demographische Entwicklung Sachsen-Anhalts im europäischen Vergleich 2000-2025. Auswertung und Interpretation der Ergebnisse der 5. Regionalisierten Bevölkerungsprognose des Statistischen Landesamts Sachsen-Anhalt und der EUROPOP 2008 von EUROSTAT. Leipzig.

Levin-Keitel, M.; Lelong, B.; Thaler, T. (2017): Zur Darstellung von Macht in der räumlichen Planung - Potenziale und Grenzen der Methode der systemischen Aufstellung. In: Raumforschung und Raumordnung I Spatial Research and Planning 75, 1, 31-44. doi: 10.1007/s13147-016-0443-2

Liebmann, H. (2010): Zivilgesellschaft unter Schrumpfungsbedingungen. In: Becker, E.; Gualini, E.; Runkel, C.; Graf Strachwitz, R. (Hrsg.): Stadtentwicklung, Zivilgesellschaft und bürgerschaftliches Engagement. Stuttgart, 71-84. = Maecenata-Schriften 6.

Mai, R.; Swiaczny, F. (2008): Demographische Entwicklung - Potenziale für Bürgerschaftliches Engagement. Wiesbaden. = Materialien zur Bevölkerungswissenschaft 126.

Mayer, M. (2005): Das Konzept des Sozialkapitals in der stadtpolitischen Diskussion. In: Informationen zur Raumentwicklung 9/10, 589-597.

McGarty, C.; Bliuc, A.-M.; Thomas, E. F.; Bongiorno, R. (2009): Collective Action as the Material Expression of Opinion-Based Group Membership. In: Journal of Social Issues 65, 4, 839-857. doi: 10.1111/j.1540-4560.2009.01627.x

Miggelbrink, J.; Meyer, F. (2015): Lost in Complexity? Researching the Role of Socio-Spatial Ascriptions in the Process of Peripheralization. In: Lang, T.; Henn, S.; Ehrlich, K.; Sgibnev, W. (Hrsg.): Understanding Geographies of Polarization and Peripheralization. Basingstoke, 62-79. doi: 10.1057/9781137415080_4

Monheim, H. (2005): Attraktive Infrastruktur auch in dünn besiedelten Regionen trotz Bevölkerungsrückgangs. Einführung des Moderators. In: Strubelt, W.; Zimmermann, H. (Hrsg.): Demographischer Wandel im Raum: Was tun wir? Gemeinsamer Kongress 2004 von ARL und BBR. Hannover, 63-65. = Forschungs- und Sitzungsberichte der ARL 225.

Nadler, R. (2012): Should I stay or should I go? International migrants in the rural town of Zittau (Saxony) and their potential impact on regional development. In: European Countryside 4, 1, 57-72. doi: 10.2478/v10091-012-0014-7

Neergard, U. (2009): Services of general economic interest: The nature of the beast. In: Krajewski, M.; Neergaard, U.; van de Gronden, J. (Hrsg.): The changing legal framework for services of general interest in Europe - between competition and solidarity. Den Haag, 17-50. doi: 10.1007/978-90-6704-725-8_2

Neu, C. (2007): Bürgerschaftliches Engagement als Erfolgsfaktor für den ländlichen Raum. In: UNESCO heute 54, 2, 34-37.

Perrons, D. (2012): Regional performance and inequality: linking economic and social development through a capabilities approach. In: Cambridge Journal of Regions, Economy and Society 5, 1, 15-29. doi: $10.1093 / \mathrm{cjres} / \mathrm{rsr} 033$

Pollack, D. (2004): Zivilgesellschaft und Staat in der Demokratie. In: Klein, A.; Kern, K.; Geißel, B.; Berger, M. (Hrsg.): Zivilgesellschaft und Sozialkapital. Herausforderungen politischer und sozialer Integration. Wiesbaden, 23-40. = Bürgergesellschaft und Demokratie 14. doi: 10.1007/978-3-322-80963-6_3

PoSCoPP - Research Group Production of Space in the Context of Polarization and Peripheralization (2015): Understanding New Geographies of Central and Eastern Europe. In: Lang, T.; Henn, S.; Ehrlich, K.; Sgibnev, W. (Hrsg.): Understanding Geographies of Polarization and Peripheralization. Basingstoke, 1-21. doi: 10.1057/9781137415080_1

Priller, E. (2010): Stichwort: Vom Ehrenamt zum zivilgesellschaftlichen Engagement. In: Zeitschrift für Erziehungswissenschaft 13, 2, 195-213. doi: 10.1007/s11618-010-0125-8

Prognos/Generali (2009): Engagementatlas 2009. Daten, Hintergründe, Volkswirtschaftlicher Nutzen. Berlin/Aachen.

Putnam, R. D. (1993): Making democracy work. Civic traditions in modern Italy. Princeton.

Putnam, R. D. (2000): Bowling alone. The collapse and revival of American community. New York.

Reim, D.; Schmithals, J. (2008): Kooperationsnetzwerke und Bürgerschaftliches Engagement als Erfolgsfaktoren für ostdeutsche Kommunen. In: Barlösius, E.; Neu, C. (Hrsg.): Peripherisierung eine neue Form sozialer Ungleichheit? Berlin, 75-82. = Materialien der Berlin-Brandenburgischen Akademie der Wissenschaften 21.

Reimer, M. (2016): Planungskultur - Eine Bestandsaufnahme. In: disP - The Planning Review 52, 4, 18-29. doi: 10.1080/02513625.2016. 1273661

Romeu Gordo, L.; Vogel, C. (2017): Geldzahlungen, Sachzuwendungen und Kostenerstattung im freiwilligen Engagement. In: Simonson, J.; Vogel, C.; Tesch-Römer, C. (Hrsg.): Freiwilliges Engagement in Deutschland. Der Deutsche Freiwilligensurvey 2014. Wiesbaden, 377-412. doi:10.1007/978-3-658-12644-5_15

Schubert, H.-J. (2002): Demokratie in der Kleinstadt. Eine empirische Studie zur Motivation lokalpolitischen Handelns. Wiesbaden. doi: 10.1007/978-3-322-89960-6

Siebel, W. (2010): Planende Verwaltung und zivile Gesellschaft. In: Becker, E.; Gualini, E.; Runkel, C.; Graf Strachwitz, R. (Hrsg.): Stadtentwicklung, Zivilgesellschaft und bürgerschaftliches Engagement. Stuttgart, 25-38. = Maecenata-Schriften 6.

Simonson, J.; Vogel, C. (2017): Organisationale Struktur des freiwilligen Engagements und Verbesserungsmöglichkeiten der Rahmenbedingungen. In: Simonson, J.; Vogel, C.; Tesch-Römer, C. (Hrsg.): Freiwilliges Engagement in Deutschland. Der Deutsche Freiwilligensurvey 2014. Wiesbaden, 523-548. doi: 10.1007/9783-658-12644-5_21

Simonson, J.; Ziegelmann, J. P.; Vogel, C.; Tesch-Römer, C. (2017): Zusammenfassung. In: Simonson, J.; Vogel, C.; Tesch-Römer, C. (Hrsg.): Freiwilliges Engagement in Deutschland. Der Deutsche Freiwilligensurvey 2014. Wiesbaden, 21-27. doi: 10.1007/978-3658-12644-5_1

Sittler, L. (2015): Wesentliche Ergebnisse und Handlungsempfehlungen. In: Generali Zukunftsfonds (Hrsg.): Generali Engagementatlas 2015. Köln, 8-9.

SMS - Sächsisches Staatsministerium für Soziales (2005): Bürgerschaftliches Engagement in Sachsen 2004. Dresden.

Steinführer, A. (2015): Bürger in der Verantwortung. Veränderte Akteursrollen in der Bereitstellung ländlicher Daseinsvorsorge. In: Raumforschung und Raumordnung 73, 1, 5-16. doi: 10.1007/s13147-014-0318-3

Steinführer, A.; Küpper, P.; Tautz, A. (2012): Gestaltung der Daseinsvorsorge in alternden und schrumpfenden Gemeinden - Anpassungs- und Bewältigungsstrategien im Harz. Braunschweig. = Landbauforschung Sonderheft 367.

Swiaczny, F. (2010): Implications of Demographic Change for Civil Society in Germany. In: Journal of Population Research 27, 3, 193-211. doi: 10.1007/s12546-010-9041-2

Wade, M. (2015): Mikrokosmos Stadtviertel. Lokale Partizipation und Raumpolitik. Bielefeld.

Westle, B.; Gabriel, O. W. (2008): Sozialkapital. Eine Einführung. Baden-Baden.

Wiest, K.; Leibert, T. (2013): Wanderungsmuster junger Frauen im ländlichen Sachsen-Anhalt - Implikationen für zielgruppenorientierte Regionalentwicklungsstrategien. In: Raumforschung und Raumordnung 71, 6, 455-469. doi: 10.1007/s13147-013-0257-4 
Wiest, K.; Leibert, T.; Johansson, M.; Rauhut, D.; Ponnikas, J.; Mustonen, V.; Timár, J.; Velkey, G.; Nagy, T.; Kugler, J.; Györffy, I.; Nagy, Z.; Kocziszky, G. (2011): SEMIGRA - Selective Migration and unbalanced Sex Ratio in Rural Regions. Interim Report. Leipzig.

Winkler-Kühlken, B. (2003): Voneinander lernen - Bevölkerungsrückgang und Strukturanpassung in ländlichen Regionen Europas. In: Informationen zur Raumentwicklung 12, 779-787.
Wolff, M.; Leibert, T. (2016): Deutschlands neue Raummuster - Bevölkerungsentwicklungen auf Gemeindeebene 1990 bis 2014. In: Nationalatlas aktuell 10 vom 27. Mai 2016. http://aktuell. nationalatlas.de/Bevoelkerungsentwicklung.3_05-2016.0.html (22.08.2017).

Wollmann, H. (2013): Stadt im Blick der Kommunalwissenschaft. In: Mieg, H. A.; Heyl, C. (Hrsg.): Stadt. Ein interdisziplinäres Handbuch. Stuttgart, 174-184. 